\title{
Success of transdisciplinary science requires monodisciplinary support
}

\author{
Douglas S. Richardson
}

Received: 9 March 2016 / Accepted: 14 March 2016/Published online: 22 March 2016

C) Springer Science+Business Media Dordrecht 2016

Traditionally, academics applied to, and were welcomed into, a singular department at a university or college. This department often had its own building that sat isolated from the others on campus creating literal walls between disciplines. It was rare to see biologists interact with physicists, or chemists talk to mathematicians and even rarer to see basic scientists interact with applied scientists or fellow faculty members in the humanities. However, when these interactions did occur, often great science was the result. Today, many of these traditional academic departments exist in name only or have been disbanded all together. In their place, centers and programs that unite principal investigators with a wide range of backgrounds and expertise have arisen as the new standard of transdisciplinary research on campus.

One of the most positive impacts of transdisciplinary science has been the interactions of engineers and physicists with the various '-ologists' (biologists, toxicologists, physiologists, etc.). These cooperative research efforts have resulted in technological advances that are facilitating discoveries that were previously thought

D. S. Richardson $(\bowtie)$

Department of Molecular and Cellular Biology, Harvard University, Cambridge, MA, USA

e-mail: drichardson@fas.harvard.edu

D. S. Richardson

Harvard Center for Biological Imaging, Harvard University, Cambridge, MA, USA impossible. One example of technology developed through the collaboration of transdisciplinary researchers is the recent advances in light microscopy sample preparation and instrumentation. Sub-fields such as super-resolution (Eggeling et al. 2015), lightsheet (Reynaud et al. 2008), tissue clearing (Richardson and Lichtman 2015), and high-content screening (Zanella et al. 2010) have the potential to accelerate biomedical research, but they are not without their barriers to use.

Historically, there existed a subset of '-ologists' who were self-described microscopists. However, in today's transdisciplinary environment, this title is rarely applied. A life-scientist needs to be a microscopist, a geneticist, a cell biologist, a pharmacologist, a behaviorist, and a toxicologist all at the same time.

Data acquisition in each of these sub-fields is driven by a number of core technologies that are constantly, and now quite rapidly, evolving. Although these systems are highly complex and technical, they are often described by manufacturers as 'turn-key' instruments with intuitive software and controls. In fact, many scientists can sit down at a new piece of equipment and acquire data in a matter of minutes or hours, but is this data useful?

Learning to drive a car is a skill that is easily learned by most people. Within a few hours, a new driver can reliably pilot a car down a road that many other people have driven before. However, this does not qualify someone to participate in high level motorsports or drive the vehicle into previously unexplored areas. Knowledge of the technology behind how a car functions and its limitations are all required to become an elite driver. 
So, it is with science. To develop an assay that can provide accurate, reliable, and reproducible data, a high level of technical knowledge regarding the scientific instrument to be used must first be acquired. This knowledge of how the system works, its abilities and its limits will influence all aspects of the experiment from initial design to data analysis.

For example, examining the intricate spatial relationships of the various components of the nuclear pore complex (NPC) is impossible with a widefield or confocal microscope. No matter how bright the signal, or how sensitive the detectors, the molecules of interest lie too close to one another-below the diffraction limit of a light microscope. A researcher needs to understand this limit and have the knowledge to select a superresolution light microscopy technique or utilize the higher resolving power of electron microscopy to investigate spatial relationships within the NPC.

Therefore, the ideal transdisciplinary scientist is not only a master of many life science -ologies but also the applied science 'techn' -ologies. The truth is very few scientists, if anyone, can be expected to be knowledgeable in all of these areas. Therefore, as we press forward with our desire to train transdisciplinary scientists, let us not forget that they will still need support and collaboration from a cohort of monodisciplinary specialists who have an extensive knowledge of their subject and its associated technologies. Only with monodisciplinary specialists working in support of multiple transdisciplinary research groups will science be able to move forward most efficiently.

\section{References}

Eggeling C, Willig KI, Sahl SJ, Hell SW. Lens-based fluorescence nanoscopy. Q Rev Biophys. 2015;48:178-243.

Reynaud EG, Krzic U, Greger K, Stelzer EH. Light sheetbased fluorescence microscopy: more dimensions, more photons, and less photodamage. HFSP J. 2008;2:26675.

Richardson DS, Lichtman JW. Clarifying tissue clearing. Cell. 2015;162:246-57.

Zanella F, Lorens JB, Link W. High content screening: seeing is believing. Trends Biotechnol. 2010;28:237-45. 